\title{
Location Fingerprint Positioning Technology using Bat Algorithm
}

\author{
Song Lixin ${ }^{1}$, Zhou Chuanbin ${ }^{1}$ and Pei Heng ${ }^{2}$ \\ ${ }^{1}$ College of Electric and Electronic Engineering, \\ Harbin University of Science and Technology, China \\ ${ }^{2}$ Navigation and Control Technology Research Institute of China \\ North Industries Group Corporation, China \\ lixins99@aliyun.com
}

\begin{abstract}
A location fingerprint positioning technology method is proposed based on bat algorithm to improve the defect of traditional indoor location fingerprint positioning technology. These shortcomings include offline heavy workload, limited accuracy and poor robustness. The fingerprint database can generate using the middle point interpolation method and the method that channel attenuation model which instead of offline training stage. This stage realize the function of timing automatic databased updates. After that the database combines $K$ nearest neighbor algorithm with bat algorithm in the stage of matching algorithm to realize the positioning function. Compared with the traditional method, location fingerprint positioning technology using bat algorithm reduces the overall positioning of workload and rapidly respond to the effect of the changing environment. Finally, localization performance test is carried out under the given simulation environment. The result indicates that this method improves the average precision than other algorithms about $23.14 \%$, the vast most of the blind node position error range within 1.5 meters, which shows the advantages of positioning accuracy, robustness and adaptation to the changing environment.
\end{abstract}

Keywords: Location fingerprint positioning; Middle point interpolation; Channel attenuation model; Bat algorithm

\section{Introduction}

The wireless signal easy has the phenomenon such as reflection diffraction and scattering because of complex indoor environment. These phenomena will bring the wireless signal propagation multipath effect, so that the new localization algorithm was studied. Nowadays, the mainly indoor wireless location technology includes Wi-Fi, ZigBee, Ultra-Wideband (UWB), Bluetooth, Radio Frequency Identification (RFID), etc [1-5]. Comparing with the indoor wireless location technology, the wireless location method is divided into two parts, the localization algorithm based on the distance measurement and the localization algorithm based on the range-free measurement. The localization algorithm based on the distance measurement mainly includes Time-OfArrival (TOA), Time-Difference-Of-Arrival (TDOA), Angel-Of-Arrival (AOA) and the localization algorithm based on the Received Signal Strength Indication (RSSI) [5, 6]. The localization algorithm based on the range-free measurement, by the way, includes centroid localization algorithm, Distance-Vector-Hop (DV-Hop), Amorphous, A. Punctate Ink Toxin (APIT) [7, 8]. Location fingerprinting positioning technology is an indoor wireless localization algorithm based on RSSI. After the impact of the indoor environment of the multipath, the radio waves form their own unique multipath structure in each position, and then, this structure can be saw as "fingerprints" of this 
position. Location fingerprinting position technology is divided into two stages: the stages of offline and online.

- Offline Phase

The main work of the offline phase is to set some fingerprints location points in the designated area. Then using the wireless devices to collect the RSSI from the each fingerprint location point and set these RSSI as its position fingerprint. After that, these RSSI and the coordinates where receive these RSSI builds a fingerprint database. The large number of fingerprint point position we set, the more precise locating results we get, but these bring the more workload $[9,10]$.

- Online Phase

In the online phase the blind nodes take the RSSI it collect to traverse the fingerprint database so that it can use the special algorithm to match the RSSI and the location coordinate from fingerprint database. The commonly used matching algorithm in fingerprint localization technology includes Nearest Neighbor (NN), K-Nearest Neighbor (KNN), Weighted Nearest Neighbor (WKNN), Bayesian probability algorithm, BP Neural Network algorithm, etc [11, 12]. K-Nearest Neighbor algorithm [13] is improved on the basis of Nearest Neighbor algorithm. The blind node uses the $S=\left(s_{1}, s_{2}, s_{3} \cdots, s_{n}\right)$ that it collected to traverse the fingerprint database and selects the most closely $\mathrm{K}(K \geq 2)$ fingerprint location points. After calculating the average of the $\mathrm{K}$ fingerprint location points' coordinates and set this average coordinate as the coordinate of the blind node.

Bat algorithm (BA) put forward by Yang is a new kind of swarm intelligence algorithm which simulates the bat echolocation behavior $[14,15]$. Comparing with the intelligent algorithms such as Particle Swarm Optimization, Genetic Algorithm, and the bat algorithm can dynamically control the mutual transformation between the global search and the local search process. In this way, bat algorithm can avoid falling into local optimal, and it has the better convergence [16, 17].

Shadowing model [18] is a propagation model which can show the wireless signal strength attenuation process, its expression is:

$R S S I=A-10 \times n \times \log _{10}(d)$

Among them: $n$ shows the signal propagation constants, $d$ describes the distance between the receiver and the transmitter of the signal, $A$ shows the $1 \mathrm{~m}$ distance of anchor node's RSSI, especially, $A$ an $n$ of the model can reset according to the specific experimental environment. In view of the large workload of the traditional fingerprint positioning technology offline phase and the poor positioning accuracy and positioning stability, this thesis put forward a new location fingerprint positioning based on bat algorithm.

\section{Location Fingerprint Positioning based on Bat Algorithm}

\subsection{Overall Design of the Algorithm}

The offline phase of the traditional location fingerprint positioning technology using the manual way to set the fingerprint points and collect the RSSI to build the fingerprint database. Location fingerprint positioning technology relies on the indoor environment. The original established fingerprint database cannot objectively reflect the distribution state of the signal strength when the indoor environment changes or moving the anchor nodes. This phenomenon increases the positioning error so that the fingerprint database should be rebuilt. However, this method further increases the workload. The location fingerprint positioning technology based on bat algorithm has no offline phase. Both of 
the establishment and regularly update of the fingerprint database and the positioning of blind nodes are all completed in the online phase. The total diagram of location fingerprint positioning based on bat algorithm technology shows in Figure 1.

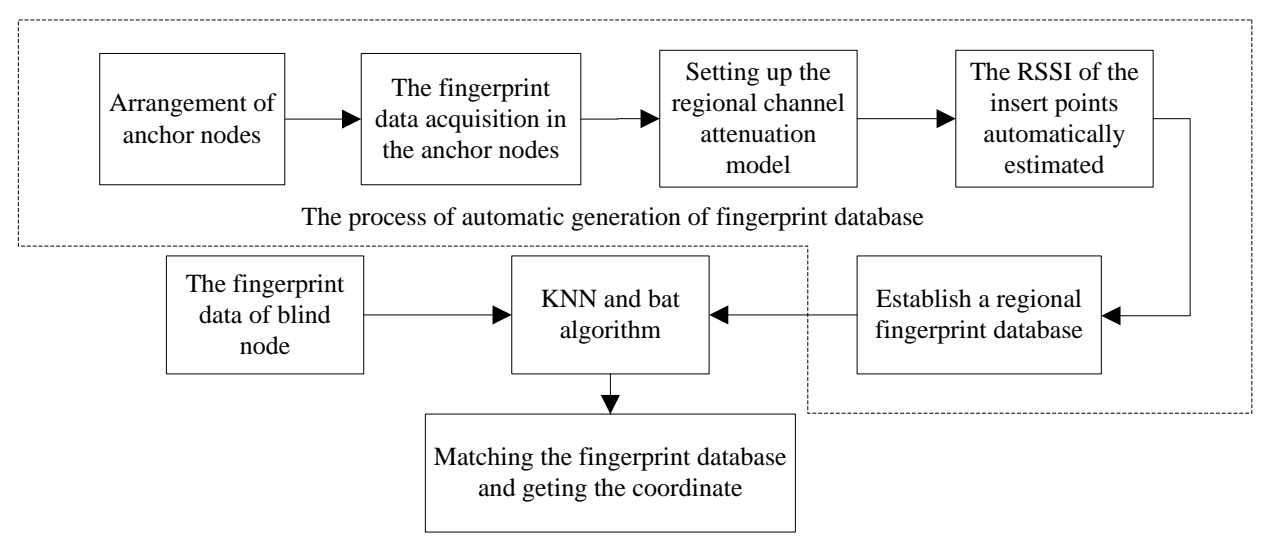

\section{Figure 1. The Total Diagram of Location Fingerprint Positioning based on Bat Algorithm Technology}

Specific design of the algorithm:

1. Firstly the $\mathrm{N}(N \geq 3)$ anchor nodes which known its position coordinates were evenly deployed in the location area. Then the wireless devices get the RSSI of the each anchor node to build a part of the fingerprint database. The deployed anchor nodes should be uniform as far as possible to facilitate subsequent generation fingerprint points of fingerprint database distributed evenly.

2. This thesis chooses the widely used and comprehensive model named Shadowing to set up the model of the regional channel attenuation. The received signal strength A and the signal propagation constants $\mathrm{n}$ of the Shadowing model can be estimated in the specific environment. The value of the parameter of the Shadowing model can be updated regularly and each anchor node uses the received signal strength and its own position coordinates to verify its channel attenuation model.

3. The fingerprint location point inserted into the midpoint of any two anchor node. And then, this point automatically calculates the RSSI by using the channel attenuation model. After that, the complete fingerprint database built combines the RSSI and its location coordinate.

4. The blind node collects the RSSI from each anchor node to form its fingerprint feature data. Then, the blind node uses the KNN algorithm and the bat algorithm to traverse the fingerprint database to find the best position coordinate.

\subsection{Location Fingerprint Positioning based on Bat Algorithm Technology}

2.2.1. Bat Algorithm: Bat algorithm is a random search algorithm which simulate bat to detect prey and avoid obstacles in the nature. Bat random flies in a speed of $v_{i}$ in the position of $x_{i}$ and uses a fixed frequency $f_{\min }$, variable wavelength $\lambda$ and the volume $A$ to search the prey. The bat can adjusts its wavelength of the transmitted pulse and pulse firing rate $r$ according to the distance of the bat and the prey. Among them the volume interval change of $\mathrm{A}$ is $\left[A_{\min }, A_{\max }\right]$.

2.2.2. Fitness Function Design of Bat Algorithm: The bat algorithm uses the fitness function to measure the merits of the bat position so that the speed and position of the 
bat, in iteration process, needs to update. The goal of wireless sensor positioning is to reduce the positioning error.

The location fingerprint positioning based on bat algorithm technology is a method that converts the node location problem to the constrained optimization problem. There are two kinds of nodes in the fingerprint database. One is the anchor nodes set in the location environment and the other is the nodes which are in the middle of any two anchor nodes. Because of the position coordinates known, so the distance between the nodes in the fingerprint database and the blind node can be calculated. Hence, the fitness function of the bat algorithm is:

$$
f(i)=\sum_{i=1}^{K} \sqrt{\left(x_{i}-x\right)^{2}+\left(y_{i}-y\right)^{2}-d_{i}^{2}}
$$

Among them, $\mathrm{K}$ is the number of fingerprint position points which are the nearest to blind node in the fingerprint database and $d_{i}$ means the distance between the receiver and transmitter of signal.

\subsubsection{Process of the Location Fingerprint Positioning based on Bat Algorithm:}

1. The $\mathrm{N}$ anchor nodes set in the location area, and then, some fingerprint location points set in the area by using the middle point interpolation method. The fingerprint database contains those nodes and records the RSSI and the location coordinates according the channel attenuation model. The number of the whole fingerprint database is

num $=C_{N}^{2}+N$

2. The method select the $\mathrm{K}$ fingerprint location points from the fingerprint databased whose RSSI is closest to the RSSI of the blind node $p(x, y)$.

3. The constraint condition of the blind node $p(x, y)$ got from the distance between the $\mathrm{K}$ fingerprint location points and the blind node and the formula1. Formula 2 shows the constraint relation.

4.This stage initializes the related parameters of the bat algorithm, mainly includes the number $m$ of the individuals in the population, pulse emissivity $r$, volume $A$, the frequency range of the searching pulse $\left[f_{\min }, f_{\max }\right]$, the attenuation coefficient of the volume $\alpha$, the enhanced coefficients of the searching frequency $\gamma$, the largest number of iterations iter_max and maximum pulse firing rate $r_{0}$.

5. Random initialization of the location of the bat $x_{i}$. This bat means the blind node $p(x, y)$, and find out the current best solution $x^{*}$ according the fitness function.

6. The speed, location coordinate and the frequency of searching pulse of the bat algorithm all need updated based on the initial value. These parameters of bat algorithm, in the process of evolution, are updated by the following formula:

$f_{i}=f_{\text {min }}+\left(f_{\text {max }}-f_{\text {min }}\right) \times \beta$

$v_{i}^{t}=v_{i}^{t-1}+\left(x_{i}^{t}-x^{*}\right) \times f_{i}$

$x_{i}^{t}=x_{i}^{t-1}+v_{i}^{t}$

Among them: $\beta$ is a random variable of uniform distribution on $\beta \in[0,1], f_{i}$ is the searching pulse frequency of bat $i, v_{i}^{t}$ and $v_{i}^{t-1}$ respectively shows the speed of the bat 
$i$ in the time $t$ and $t-1, x_{i}^{t}$ and $x_{i}^{t-1}$ respectively shows the location coordinate of the bat $i$ in the time $t$ and $t-1, x^{*}$ is the optimal solution of all current bat.

7. This algorithm set a uniformly distributed random number $\operatorname{rand}_{1}$, if $\operatorname{rand}_{1}>r_{i}$, the current optimal solution $x^{*}$ need be random disturbed, so that there is a new solution. However, the solution should be deleted if it out of the range of location area.

8. Next, the method set another uniformly distributed random number rand $_{2}$, the new solution generated from stage 7 should be accepted if rand $_{2}<A_{i}$ and $f\left(x_{i}\right)<f\left(x^{*}\right)$. Then the parameter $r$ and $A_{i}$ updated by the following formulas.

$A_{i}^{t+1}=\alpha A_{i}^{t}$

$r_{i}^{t+1}=r_{0} \times\left[1-e^{-\gamma t}\right]$

Among them $\alpha$ is the volume attenuation coefficient and usually take on the constant between $[0,1], \gamma$ is the enhance coefficient of searching frequency and it usually greater than zero, $A_{i}^{t}$ shows the volume of bat $i$ in the time $t, r_{i}^{t+1}$ is the pulse emissivity of the bat $i$ in the time $t+1$.

9. Sort the fitness value of all the bats and find out the current optimal solution and optimal value.

10. Repeat step 5 to 9 , until complete set the maximum number of iterations.

11. Output global optimal fitness value corresponding to the location of the bat, that is, the best location coordinate $(x, y)$ of the blind node $p(x, y)$.

The whole algorithm flow chart as shown in Figure 2.

2.2.4. Time Complexity of the Algorithm: The time complexity of algorithm of the location fingerprint positioning technology based on the bat algorithm mainly depends on the baa population and the iterations of bat algorithm. Assuming that the bat population is $m$ and the maximum iterations of the bat algorithm is iter_max. The time complexity is:

$O\left(m \times i t e r \_m a x\right)$ 


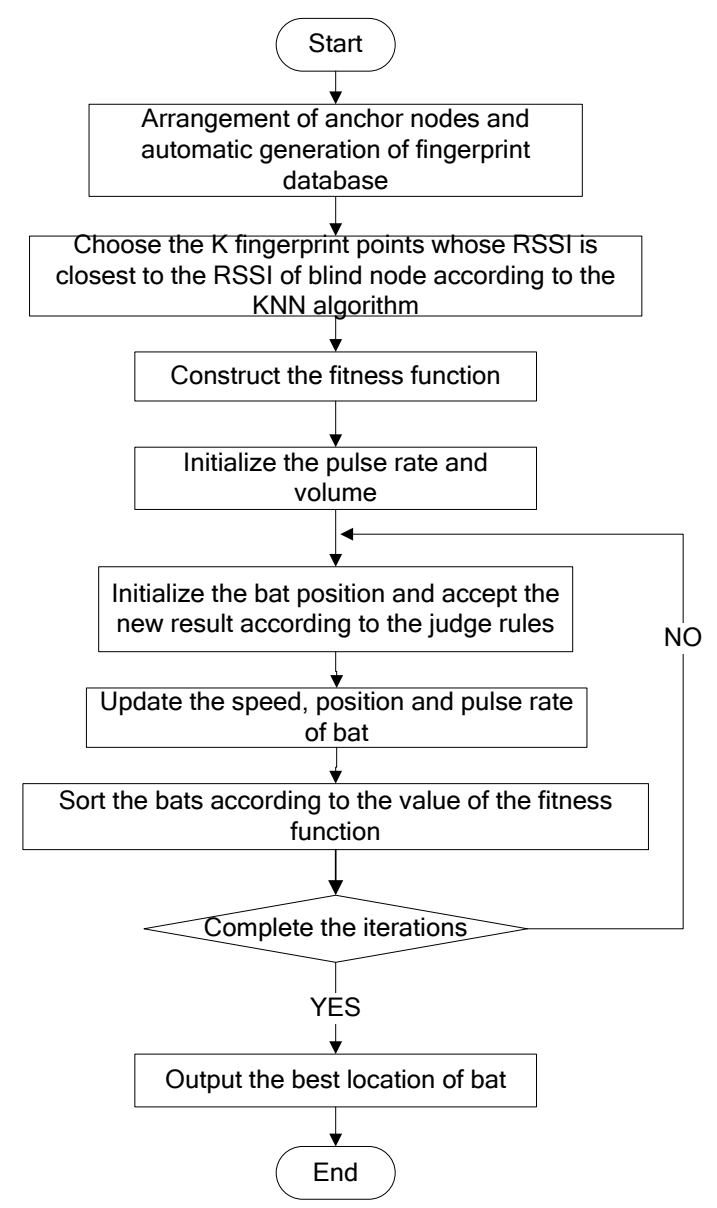

Figure 2. The Whole Algorithm Flow Chart

\section{Simulation Experiment}

\subsection{Simulation Scene}

The location fingerprint positioning technology based on bat algorithm tested in Matlab simulation platform in order to know its practical effects. 100 nodes (including blind node only) random distribute in the area. Firstly, compare the positioning results of bar algorithm with the positioning results of NN, KNN, and WKNN in the case of offline stage to verify the superiority of the bat algorithm in the fingerprint positioning technology. The fingerprint database automatically generated in the same area (no offline stage). In this environment, compare the positioning results of bat algorithm with the positioning results of the other algorithms to verify the performance of the algorithm. The average position error and cumulative probability distribution function used as a positioning evaluation standard results.

error $=\frac{1}{M} \sum_{i=1}^{M} \sqrt{\left(x_{i}-\hat{x_{i}}\right)^{2}+\left(y_{i}-\hat{y_{i}}\right)^{2}} \times 100 \%$

Among them: $\left(x_{i}, y_{i}\right)$ and $\left(x_{i}, y_{i}\right)$ respectively shows the estimated position and the actual position of the blind node $i, M$ means the total number of blind node.

Cumulative distribution function of formula is as follows:

$F(x)=P(X \leq x)$ 
Among it, $x$ shows the difference between each blind node and its positioning result. $x=$ error .

\subsection{Result and Analysis}

1. Experiment 1 makes comparisons of the positioning error value and cumulative probability distribution function between the positioning result of bat algorithm and $\mathrm{NN}, \mathrm{KNN}, \mathrm{WKNN}(\mathrm{K}=4)$ when these all under the premise that using the location fingerprint positioning technology. Figure 3 illustrates the comparisons the cumulative probability distribution function of the various algorithm.

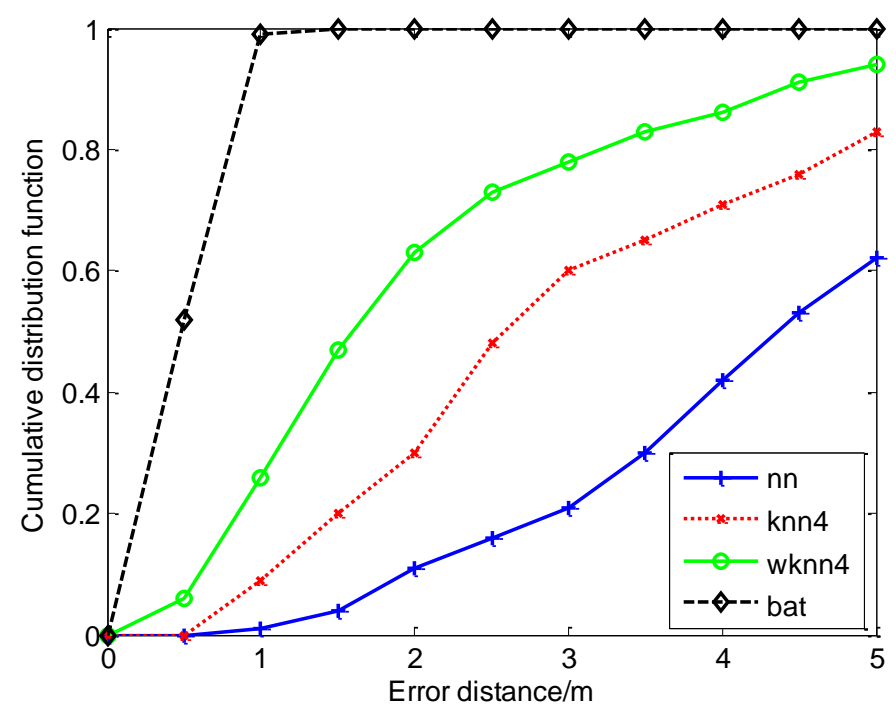

Figure 3. The Cumulative Probability Distribution Function

The positioning error range of bat algorithm compares with other three matching algorithm is small from the cumulative probability distribution function of Figure 1, most of the error in not more than 1.5meters. In addition, the average positioning accuracy of bat algorithm is about 1meter. Comparing with other algorithm, the average positioning accuracy is increases about $23.14 \%$. So, the bat algorithm has not only higher positioning accuracy and better robustness.

2. Experiment 2 applies the bat algorithm to the location fingerprint positioning technology of automatic generation of fingerprint database $(\mathrm{k}=4)$. There are two ways in order to make the final positioning error can achieve the experiment 1 positioning error. The first way is that the number of the fingerprint positioning of automatically generating fingerprint database can close to the number of the fingerprint database of experiment 1 . The way can realized by more anchor nodes uniform arranged and combine the nodes that set into the middle of any two anchor nodes using middle point interpolation method to build the fingerprint database. The second method is increasing the number of the bat population to reduce the positioning error under the condition of small number of anchor nodes. Although this method increases the amount of calculation, reduces the number of anchor nodes. The positioning results of experiment 1 and experiment are close because of the similar fingerprint database in the first way. The following figure illustrates the comparison of the cumulative probability distribution function of various algorithms by busing the second method. 


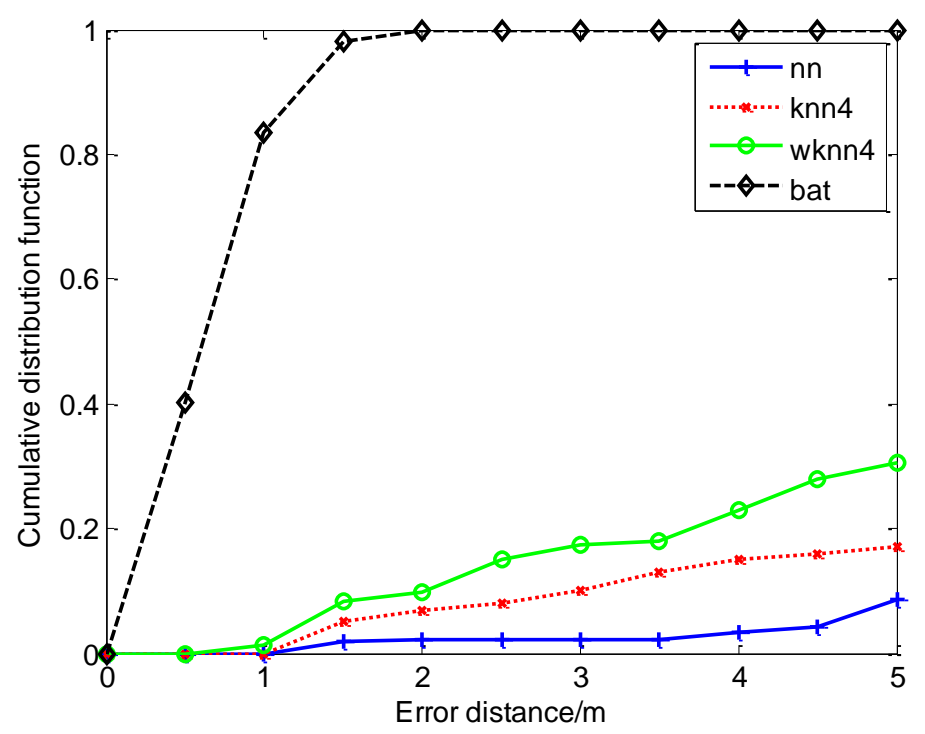

Figure 4. The Cumulative Probability Distribution Function

This experimental only set 6 anchor nodes, so the number of positioning fingerprint in the fingerprint database is 21 according to the middle point interpolation method. This method increases the number of the bat population $(\mathrm{m}=1000)$ taking the place of setting a large number of anchor nodes comparing with experiment 1 . The cumulative probability distribution function of bat algorithm is earlier arrived at 1 comparing with other algorithm from the above figure. Besides that, most of the positioning error of bat algorithm is less than 1.5 meters, therefore, bat algorithm compared with other algorithm using in the location fingerprint positioning technology has obvious advantage. The positioning error of automatic generation of fingerprint database is close to the result of the experiment 1 , from the final positioning. Both of them are all about 1 meter. Figure 5 shows the positioning result of 100 blind nodes by using the location fingerprint positioning technology based on bat algorithm.

In the $100 m \times 100 m$ location area, there are 6 anchor nodes and 100 blind nodes arranged in it from the above figure. The algorithm using the 6 anchor nodes with the channel attenuation function and the middle point interpolation method build the fingerprint database. The figure clearly shows the 100 blind nodes and its orientation position. The vast majority positioning error of the blind nodes and its orientation positon controlled in a smaller range. In this thesis, the relation between the value of fitness function of 5 blind nodes and its iterations as the experimental considerations of the bat algorithm convergence. The relationship shows in Figure 5. 


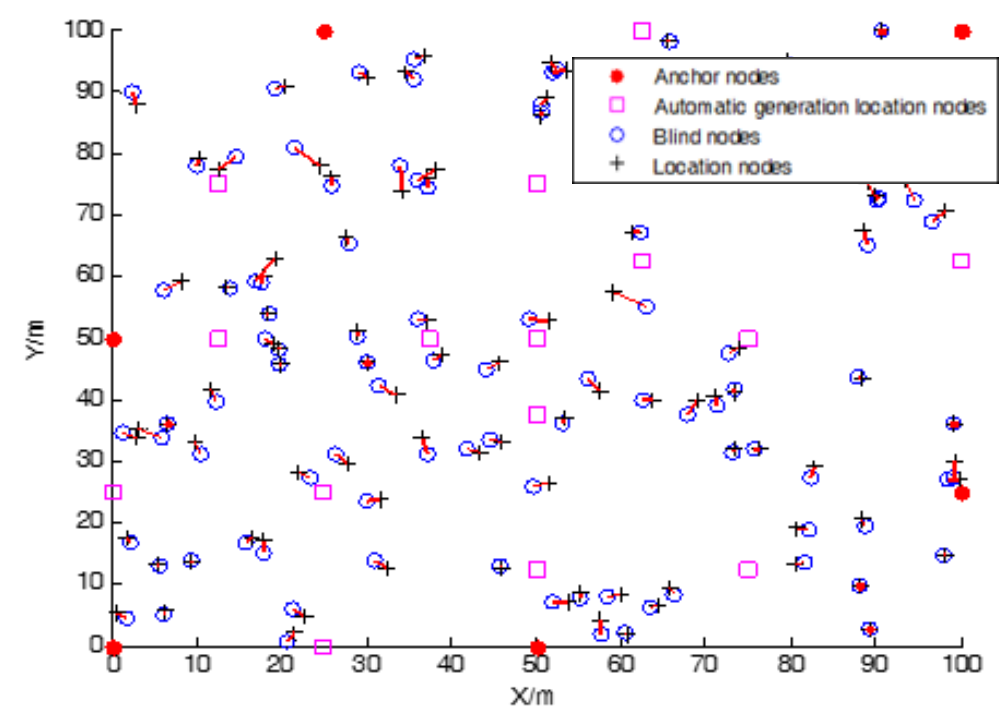

Figure 5. The Figure of Positioning Result

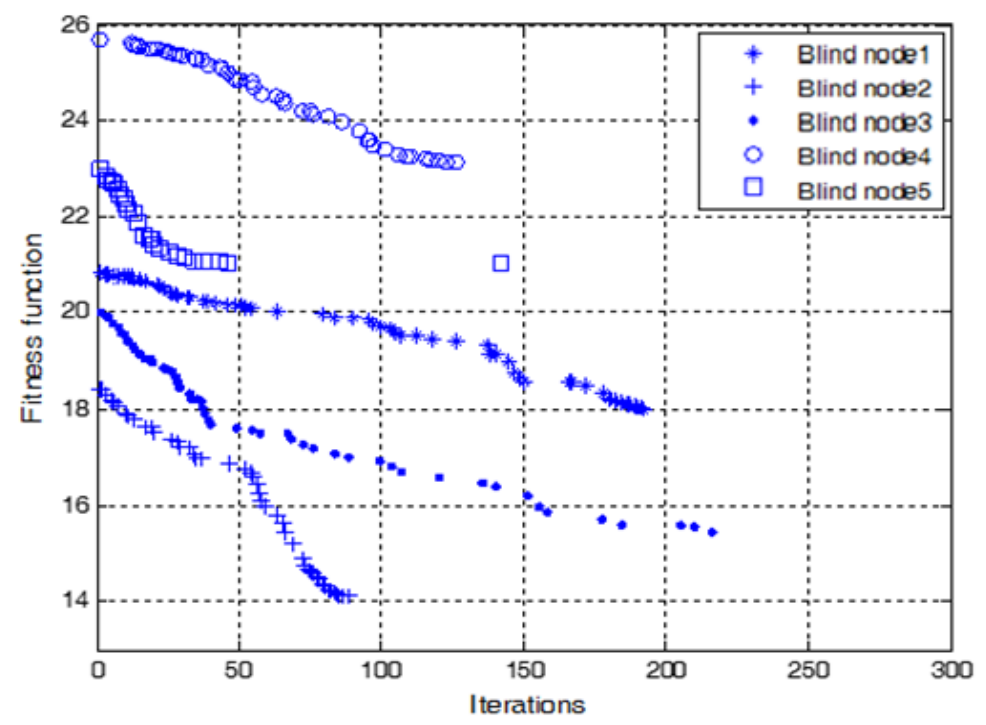

\section{Figure 6. The Relationship between the Value of Fitness Function and Iterations}

From the Figure 6, the trend of change that the value of fitness function of bat algorithm with the increasing of the iterations displays a good convergence of bat algorithm.

To sum up, the location fingerprint positioning technology based bat algorithm in both the accuracy and robustness than other algorithm has certain advantages, especially on the robustness, can well control the scope of the position error.

\section{Conclusion}

Because of the characteristics of the automatic generation of fingerprint database, the location fingerprint positioning technology based on bat algorithm is suitable for indoor localization of not very complex environment. Besides that, it can overcome the 
shortcomings that less anchor nodes and solve the problem that the offline stage of the location fingerprint positioning technology has lots of workload. Moreover, the fingerprint database can update regularly by using the channel attenuation model and the middle point interpolation method. The algorithm put up in this thesis not only can improve the positioning accuracy but also enhance the stability of positioning. The experimental results show that, comparing with other algorithms, the average positioning accuracy improved about $23.14 \%$ under the simulation environment. The location fingerprint positioning technology based on bat algorithm makes more significant contribution on the robustness because it can well control the positioning error in 1.5 meters. All in all, location fingerprint positioning technology based on bat algorithm is a good solution in indoor localization.

\section{References}

[1] Rekimoto J., Miya Ki T. and Ishizawa T., "Life Tag: Wi-Fi-based continuous location logging for life pattern analysis LoCA", (2007), pp. 35-49.

[2] Kaemarungsi K., Ranron R. and Pongsoon P., "Study of received signal strength indication in ZigBee location cluster for indoor localization Electrical Engineering/Electronics", Computer, Telecommunications and Information Technology (ECTI-CON), 2013 10th International Conference on. IEEE, (2013), pp. 1-6.

[3] Okamoto E., Horiba M. and Nakashima K., "Particle swarm optimization-based low-complexity three-dimensional UWB localization scheme Ubiquitous and Future Networks (ICUFN)", 2014 Sixth International Conf on. IEEE, (2014), pp. 120-124.

[4] Madhavapeddy A. and Tse A., "A study of Bluetooth propagation using accurate indoor location mapping UbiComp”, Ubiquitous Computing. Springer Berlin Heidelberg, (2005), pp. 105-122.

[5] Montaser A. and Moselhi O., "RFID indoor location identification for construction projects J. Automation in Construction", vol. 39, (2014), pp. 167-179.

[6] YQ Ma, "Research and implementation of positioning technology based on wireless sensor network", Beijing: Beijing University of Posts and Telecommunication, (2006).

[7] Y. Wang and JG. Lin, "Indoor location technique based on RFID J. China instrumentation", vol. 2, (2011), pp. 54-57.

[8] Virtuani A. and Fanni L., "Seasonal power fluctuations of amorphous silicon thin-film solar modules: distinguishing between different contributions J. Progress in Photovoltaics: Research and Applications", vol. 22, no. 2, (2014), pp. 208-217.

[9] Kaemarungsi K. and Krishnamurthy P., "Modeling of indoor positioning systems based on location fingerprinting INFOCOM 2004", Twenty-third Annual Joint Conference of the IEEE Computer and Communications Societies. IEEE, vol. 2, (2004), pp. 1012-1022.

[10] H. Yi, "Location fingerprint positioning technology J. Electronic technology of Shanxi", vol. 5, (2007), pp. 84-87.

[11] SW Wang, "Research on wlan indoor location method based on fingerprint J. Harbin Institute of Technology", Harbin, (2009).

[12] Q Yang, "Research on wireless indoor positioning method based on fingerprint. Hangzhou: Zhejiang University", (2011).

[13] L. Tang, YB. Xu and M. Zhou, "Research on K nearest neighbors algorithm under the indoor wlan International forum on information technology and applications", (2009).

[14] Yang X. S., "A new metaheuristic bat-inspired algorithm Nature inspired cooperative strategies for optimization (NICSO 2010)", Springer Berlin Heidelberg, (2010), pp. 65-74.

[15] Yang X. S. and Hossein Gandomi A., "Bat algorithm: a novel approach for global engineering optimization J. Engineering Computations", vol. 29, no. 5, (2012), pp. 464-483.

[16] ZB Wang, "Node localization of wireless sensor networks based on bat algorithm J. Computer Engineering and Applications", vol. 50, no. 11, (2014).

[17] AT Tan, H. Chen and BQ Wu, "Nodes localization method of wireless sensor networks based on BatDVHop algorithm J. Computer Engineering and Applications", vol. 50, no. 17, (2014).

[18] X. Pei, HQ Yu and GS Fan, "Research on mining personnel location algorithm based on ZigBee technology J. Computer Engineering and Applications”, vol. 50, no. 15, (2014), pp. 249-254. 